\title{
Exact solutions of dispersion equation for MHD waves with short-wavelength modification
}

\author{
CHEN Ling ${ }^{1,2} \&$ WU DeJin ${ }^{1 *}$ \\ ${ }^{1}$ Purple Mountain Observatory, Chinese Academy of Sciences, Nanjing 210008, China; \\ ${ }^{2}$ Graduate University of Chinese Academy of Sciences, Beijing 100012, China
}

Received August 30, 2010; accepted November 9, 2010

\begin{abstract}
Dispersive magnetohydrodynamic (MHD) waves with short-wavelength modification have an important role in transforming energy from waves into particles. In this paper, based on the two-fluid mode, a dispersion equation, including the short-wavelength effect, and its exact solution are presented. The outcome is responsible for the short-wavelength modification versions of the three ideal MHD modes (i.e. the fast, slow and Alfvén). The results show that the fast and Alfvén modes are modified considerably by the shortwavelength effect mainly in the quasi-parallel and quasi-perpendicular propagation directions, respectively, while the slow mode can be affected by the short-wavelength effect in all propagation directions. On the other hand, the dispersive modification occurs primarily in the finite $\beta$ regime of $0.001<\beta<1$ for the fast mode and in the high- $\beta$ regime of $0.1<\beta<10$ for the slow mode. For the Alfvén mode, the dispersive modification occurs from the low- $\beta$ regime of $\beta<0.001$ through the high- $\beta$ regime of $\beta>1$.
\end{abstract}

plasma, MHD waves, short-wavelength effect, dispersion relation

Citation: Chen L, Wu D J. Exact solutions of dispersion equation for MHD waves with short-wavelength modification. Chinese Sci Bull, 2011, 56: 955-961, doi $10.1007 / \mathrm{s} 11434-011-4409-\mathrm{Z}$

It is well known that there are three ideal magnetohydrodynamic (MHD) modes: the fast, slow and Alfvén waves in the low-frequency limit (much lower than the ion cyclotron frequency $\omega_{c i}$ ) and the long-wavelength limit (much longer than the ion Larmor radius $\rho_{i}$ ). When wavelengths reduce to the characteristic length scales, such as $\lambda_{e}$ and $\rho_{i}$, where $\lambda_{e}$ is the electron inertial length, the ions are free while the electrons are tightly bound to magnetic field lines because their Larmor radius is much smaller than that of the ions (i.e. $\rho_{e} \ll \rho_{i}$ ). This leads to the presence of spatial charge and the dispersion of the MHD waves. In the case of the Alfvén wave, the dispersive wave is called the kinetic Alfvén wave (KAW). In the $\mathrm{KAW}$, the perturbed electric fields have a non-zero component parallel to the background magnetic field that may cause efficient heating and acceleration of the plasma particles [14].

KAWs have been extensively discussed because of their potential importance in the particle energization of plasmas.

*Corresponding author (email: djwu@ pmo.ac.cn)
Also, they have been applied to laboratory, space and astrophysical plasmas, such as tokamak plasma heating [57], auroral electron acceleration [8-11], solar coronal plasma heating [12-14], and abnormal heating of heavy ions in the extended corona $[15,16]$. On the other hand, the other two modes, the fast and slow waves, have been given little attention. Some recent studies on the MHD turbulence in interstellar and interplanetary spaces show that the energy cascades primarily by developing small-scales structures perpendicular to the local field, with $k_{\perp} \gg k_{\|}$[17-20]. Large values of $k_{\perp}$ could be the consequence of refraction [21,22], resonant absorption [23-25], and turbulent cascade [26]. This result is supported by numerical simulations of magnetized turbulence with a dynamically strong mean field [27,28]. In situ measurements of turbulence in the solar wind and observations of interstellar scintillation also show similar evidence for the theoretical results above [29-34].

For a warm plasma with a high $\beta$ of $\beta_{i(e)} \sim 1$, which is the case for the solar wind and the magnetosheath, the compression Alfvén wave is coupled with the acoustic wave and leads 
to fast and slow magnetosonic waves. In particular, the fact that the ion thermal speed is $v_{T_{i}} \sim v_{A}$ (the Alfvén velocity) indicates that the ion gyroradius $\rho_{i}$ is comparable to the ion inertial length $\lambda_{i}$. This implies that the short-wavelength effect can considerably modify the dispersion relations of the fast and slow magnetosonic waves, as well as modifying the dispersion relation of the shear Alfvén wave, such as the case of KAWs. Some theoretical studies of MHD turbulence cascades in the solar wind show that the fluctuations in the MHD modes and their collisionless Landau damping are responsible for the steeping of the MHD turbulence spectrum at higher wavenumbers- $k_{\perp}$ (i.e. short-wavelengths) [26,35-38]. Recently, similar low-frequency phenomena has been investigated by many authors, for example, the ionospheric dynamo [39], shock arrival time [40], the response of magnetosphere to interplanetary shock $[41,42]$, suprathermal particle events [43], distribution of field-aligned current carried by Alfvén waves [44], the behavior of plasma armature [45] and MHD processes of flight vehicles [46-48].

In this paper, we derive an exact dispersion equation, based on the two-fluid model, which contains all three MHD modes with the short-wavelength modification. Furthermore, based on the dispersion equation, we present the dispersion relations, which are the versions, with short-wavelength modification, of the three modes: the fast and slow magnetosonic waves and the shear Alfvén wave. The effects of the propagation angle, the short-wavelength modification and the plasma beta on the three dispersive waves are also discussed.

\section{Dispersion equation in the two-fluid model}

In a proton-electron plasma, which is magnetized by a uniform magnetic field $\boldsymbol{B}_{0}$ along the $z$ direction, the complete set of two-fluid equations can be written as follows:

$$
\begin{gathered}
\left(\partial_{t}+\boldsymbol{v}_{i} \cdot \nabla\right) n_{i}+n_{i} \nabla \cdot \boldsymbol{v}_{i}=0, \\
\left(\partial_{t}+\boldsymbol{v}_{e} \cdot \nabla\right) n_{e}+n_{e} \nabla \cdot \boldsymbol{v}_{e}=0, \\
\left(\partial_{t}+\boldsymbol{v}_{i} \cdot \nabla\right) \boldsymbol{v}_{i}=\frac{e}{m_{i}}\left(\boldsymbol{E}+\boldsymbol{v}_{i} \times \boldsymbol{B}\right)-\frac{1}{m_{i} n_{i}} \nabla p_{i}, \\
\left(\partial_{t}+\boldsymbol{v}_{e} \cdot \nabla\right) \boldsymbol{v}_{e}=-\frac{e}{m_{e}}\left(\boldsymbol{E}+\boldsymbol{v}_{e} \times \boldsymbol{B}\right)-\frac{1}{m_{e} n_{e}} \nabla p_{e}, \\
\left(\partial_{t}+\boldsymbol{v}_{i} \cdot \nabla\right)\left(p_{i} n_{i}^{-\gamma_{i}}\right)=0, \\
\left(\partial_{t}+\boldsymbol{v}_{e} \cdot \nabla\right)\left(p_{e} n_{e}^{-\gamma_{e}}\right)=0, \\
\nabla \times \boldsymbol{E}=-\partial_{t} \boldsymbol{B}, \\
\nabla \times \boldsymbol{B}=\mu_{0} e\left(n_{i} \boldsymbol{v}_{i}-n_{e} \boldsymbol{v}_{e}\right)+\epsilon_{0} \mu_{0} \partial_{t} \boldsymbol{E},
\end{gathered}
$$

where $\boldsymbol{E}$ is the electric field, $\boldsymbol{B}$ the magnetic field, $\partial_{t}$ the partial derivative with respect to the time $t, \nabla$ the 3 -dimensional spatial gradient operator, $e$ the elementary charge, $\mu_{0}$ the permeability of free space, $\epsilon_{0}$ the permittivity of free space, $m_{e(i)}$, $n_{e(i)}, v_{e(i)}, p_{e(i)}$, and $\gamma_{e(i)}$ are the electron (ion) mass, density, velocity, thermal pressure, and polyindex, respectively, $\alpha$ is the angle between the wave vector $\boldsymbol{k}$ and the ambient magnetic field $\boldsymbol{B}_{0}$ (i.e. the $z$ axis).

We mark the unperturbed and perturbed quantities with the subscript 0 and the prefix $\delta$, respectively. For onedimensional plane waves propagating in the $x$ - $z$ plane, with wave vector $\left(k_{x}, 0, k_{z}\right)$ and the frequency $\omega$, all the perturbed quantities vary in the form: $\delta f \propto \exp \left[-i\left(\omega t-k_{x} x-k_{z} z\right)\right]$. The linearizing perturbed velocities of the ions and electrons can be obtained as follows:

$$
\begin{aligned}
\delta \boldsymbol{v}_{i \perp}-\left(\frac{\omega}{\omega_{c i}}\right)^{2} \delta \boldsymbol{v}_{i z}= & \frac{1}{B_{0}^{2}}\left(\delta \boldsymbol{E} \times \boldsymbol{B}_{0}-\frac{i \delta p_{i}}{q_{i} n_{i 0}} \boldsymbol{k} \times \boldsymbol{B}_{0}\right) \\
& -\frac{i q_{i} \omega}{m_{i} \omega_{c i}^{2}}\left(\delta \boldsymbol{E}-\frac{i \delta p_{i} \boldsymbol{k}}{q_{i} n_{i 0}}\right), \\
\delta \boldsymbol{v}_{e \perp}-\left(\frac{\omega}{\omega_{c e}}\right)^{2} \delta \boldsymbol{v}_{e z}= & \frac{1}{B_{0}^{2}}\left(\delta \boldsymbol{E} \times \boldsymbol{B}_{0}-\frac{i \delta p_{e}}{q_{e} n_{e 0}} \boldsymbol{k} \times \boldsymbol{B}_{0}\right) \\
& -\frac{i q_{e} \omega}{m_{e} \omega_{c e}^{2}}\left(\delta \boldsymbol{E}_{z}-\frac{i \delta p_{e} \boldsymbol{k}_{z}}{q_{e} n_{e 0}}\right),
\end{aligned}
$$

where the unperturbed velocity $\boldsymbol{v}_{0}=0$ and the low-frequency limit $\left(\omega \ll \omega_{c i}\right)$ have been used. We neglect the contribution of the polarization drifts of the electrons (the last parenthesis term in the transverse components of (10)) compared to that of the ions. The pressure perturbations may be obtained from eqs. (5) and (6):

$$
\delta p_{i}=\gamma_{i} T_{i 0} \delta n_{i}, \quad \delta p_{e}=\gamma_{e} T_{e 0} \delta n_{e},
$$

where the density perturbations $\delta n_{i}$ and $\delta n_{e}$ follow from eqs. (1) and (2):

$$
\delta n_{i}=n_{i 0} \frac{\boldsymbol{k} \cdot \delta \boldsymbol{v}_{i}}{\omega}, \quad \delta n_{e}=n_{e 0} \frac{\boldsymbol{k} \cdot \delta \boldsymbol{v}_{e}}{\omega} .
$$

Following $\mathrm{Wu}$ [49], for the low-frequency and the nonrelativistic cases, in which the Maxwell's displacement current term, on the right-hand side of eq. (8), may be neglected, equating Faraday's Law and Ampere's Law yields a dispersion tensor of the form $\Lambda \cdot \boldsymbol{E}=0$. The dispersion equation can then be obtained by calculating $|\Lambda|=0$ as follows:

$$
\Omega^{6}-A \Omega^{4}+B \Omega^{2}-C=0,
$$

where the coefficients

$$
\begin{gathered}
A=K_{A}+\frac{k^{2}}{k_{z}^{2}}+C ; \quad B=\frac{k^{2}}{k_{z}^{2}} K_{A}+2 C ; \\
C=\frac{\beta}{1+Q+k_{\perp}^{2} \lambda_{e}^{2}} \frac{k^{2}}{k_{z}^{2}} .
\end{gathered}
$$

In the expressions above, the parameter

$$
K_{A} \equiv \frac{1+Q+k_{\perp}^{2} \rho_{s}^{2}}{1+Q+k_{\perp}^{2} \lambda_{e}^{2}}
$$

is the coefficient of the KAW dispersion relation, $\Omega^{2}=K_{A}$ $[3,39]$, in a low- $\beta$ plasma, where $\Omega \equiv \omega /\left(k_{z} v_{A}\right)$ is the wave frequency in units of Alfvén frequency $\left(k_{z} v_{A}\right)$. The other parameters are $v_{s} \equiv \sqrt{\left(\gamma_{i} T_{0 i}+\gamma_{e} T_{0 e}\right) / m_{i}}$ : the ion acoustic 
speed, $\rho_{s} \equiv v_{s} / \omega_{c i}$ : the ion acoustic gyroradius, $\lambda_{e} \equiv c / \omega_{p e}$ : the electron inertial length, $\omega_{p e} \equiv \sqrt{n_{0} e^{2} / m_{e} \epsilon_{0}}$ : the electron plasma frequency, $\beta \equiv v_{s}^{2} / v_{A}^{2}=\beta_{i}+\beta_{e}$ : the ratio of the total thermal pressure to the magnetic pressure, $Q \equiv m_{e} / m_{i}$ : the ratio of the electron mass to the ion mass, and $k^{2}=k_{\perp}^{2}+k_{z}^{2}$ : the total wavenumber. Finally, we note that in the derivation of the dispersion eq. (13), we have used the quasi-neutrality condition $n_{i} \simeq n_{e}$.

\section{Solutions with the short-wavelength modifi- cation}

The dispersion eq. (13) can be rewritten as the standard form of the Cardan equation, that is

$$
\left(\Omega^{2}-\Omega_{0}^{2}\right)^{3}+p\left(\Omega^{2}-\Omega_{0}^{2}\right)+q=0,
$$

where

$$
p=B-3 \Omega_{0}^{4}, \quad q=-2 \Omega_{0}^{6}+B \Omega_{0}^{2}-C
$$

with the parameter $\Omega_{0}^{2}=A / 3$. When the discriminant $\Delta \equiv$ $q^{2} / 4+p^{3} / 27<0$, the Cardan eq. (16) has three real roots as follows:

$$
\begin{gathered}
\Omega_{F}^{2}=\Omega_{0}^{2}+2 R \cos \frac{\theta}{3}, \\
\Omega_{S}^{2}=\Omega_{0}^{2}+2 R \cos \frac{\theta+2 \pi}{3},
\end{gathered}
$$

and

$$
\Omega_{A}^{2}=\Omega_{0}^{2}+2 R \cos \frac{\theta+4 \pi}{3},
$$

where

$$
R=\sqrt{\Omega_{0}^{4}-\frac{B}{3}} ; \quad \theta=\arccos \frac{-q}{2 R^{3}} .
$$

In the long-wavelength limit of $k_{\perp}^{2} \lambda_{e}^{2}$ and $k_{\perp}^{2} \rho_{s}^{2} \ll 1$, one has

$$
A \cong 1+(1+\beta) \frac{k^{2}}{k_{z}^{2}}, \quad B \cong(1+2 \beta) \frac{k^{2}}{k_{z}^{2}}, \quad C \cong \beta \frac{k^{2}}{k_{z}^{2}},
$$

and $K_{A} \cong 1$. As a consequence, from eqs. (18)-(20), the three roots of the Cardan eq. (16) in the long-wavelength limit are

$$
\begin{aligned}
& \Omega_{F}^{2} \cong \frac{1+\beta}{2}\left[1+\sqrt{1-\frac{4 \beta}{(1+\beta)^{2}} \frac{k_{z}^{2}}{k^{2}}}\right] \frac{k^{2}}{k_{z}^{2}}, \\
& \Omega_{S}^{2} \cong \frac{1+\beta}{2}\left[1-\sqrt{1-\frac{4 \beta}{(1+\beta)^{2}} \frac{k_{z}^{2}}{k^{2}}}\right] \frac{k^{2}}{k_{z}^{2}},
\end{aligned}
$$

and

$$
\Omega_{A}^{2} \cong 1 \text {. }
$$

These are the dispersion relations for the ideal MHD modes (i.e. the fast and slow magnetosonic waves and the shear Alfvén wave). Therefore, the three roots, in eqs. (18)-(20), of the Cardan eq. (16) are the modified versions, including the short-wavelength effects, of the dispersion relations for the three ideal MHD waves.

Figure 1 plots the three roots in eqs. (18)-(20) versus the propagation angle $\alpha$ for the fixed parameters $k_{\perp} \rho_{s}=0.8$ and $\beta=0.5$. From the top down, the curves correspond to the fast, Alfvén and slow modes, respectively. For the sake of comparison, the corresponding ideal MHD solutions are presented as dashed lines in Figure 1.

From Figure 1, we see that the fast and Alfvén modes are considerably modified by the short-wavelength effect in the quasi-parallel and quasi-perpendicular directions, respectively. The slow mode, however, obviously departs from its ideal MHD version from $\alpha=0^{\circ}$ through $\alpha=90^{\circ}$ due to the short-wavelength effect. The parallel phase speed of the fast mode increases by a factor of $\sim 2$ in the quasiparallel direction of $\alpha<20^{\circ}$ and that of the Alfvén mode increases by a factor of $\sim 1.5$ in the quasi-perpendicular direction of $\alpha>50^{\circ}$. For the slow mode, the parallel phase speed decreases by a factor of $\sim 2$ in the quasi-parallel direction of $\alpha<20^{\circ}$ and a slightly lower factor of $\sim 1.5$ in the quasi-perpendicular direction of $\alpha>50^{\circ}$ due to the shortwavelength effect.

Figure 2 shows the dependence of the dispersion relations for the fast, Alfvén and slow modes (from the top down) on the perpendicular wavenumber $\left(k_{\perp} \rho_{s}\right)$ for the quasi-parallel ( $\alpha=15^{\circ}$ for the left column) and quasi-perpendicular ( $\alpha=$ $60^{\circ}$ for the right column) propagating cases. The fixed parameter $\beta=0.5$ has been used and the dashed lines plot the corresponding ideal MHD solutions for the sake of comparison.

From Figure 2, we have found, for all cases, the modification of the dispersion relations to be negligibly small
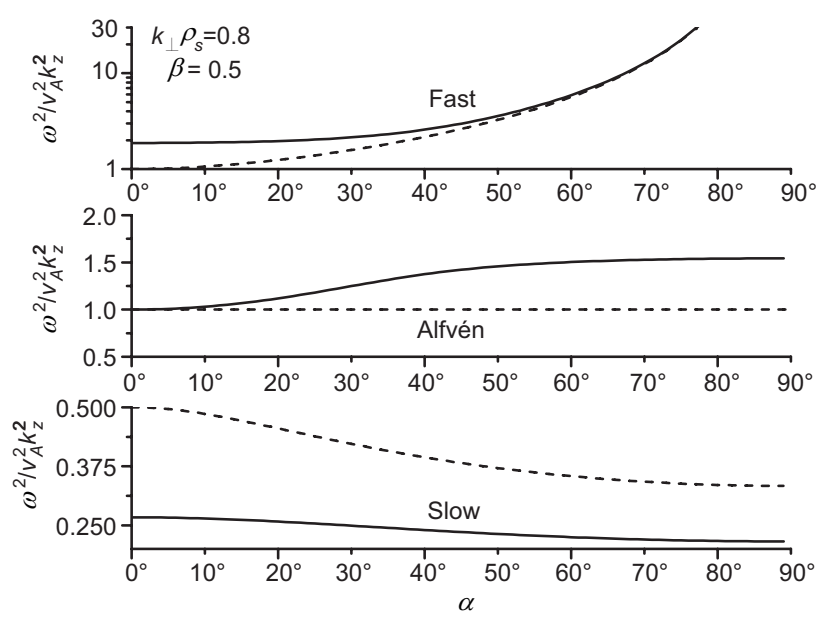

Figure 1 The dispersion relations versus the propagation angle. Panels, from the top down, present the fast, Alfvén and slow modes, respectively, where the dashed lines plot the corresponding idea MHD solutions, where the parameters $k_{\perp} \rho_{s}=0.8$ and $\beta=0.5$ have been used. 

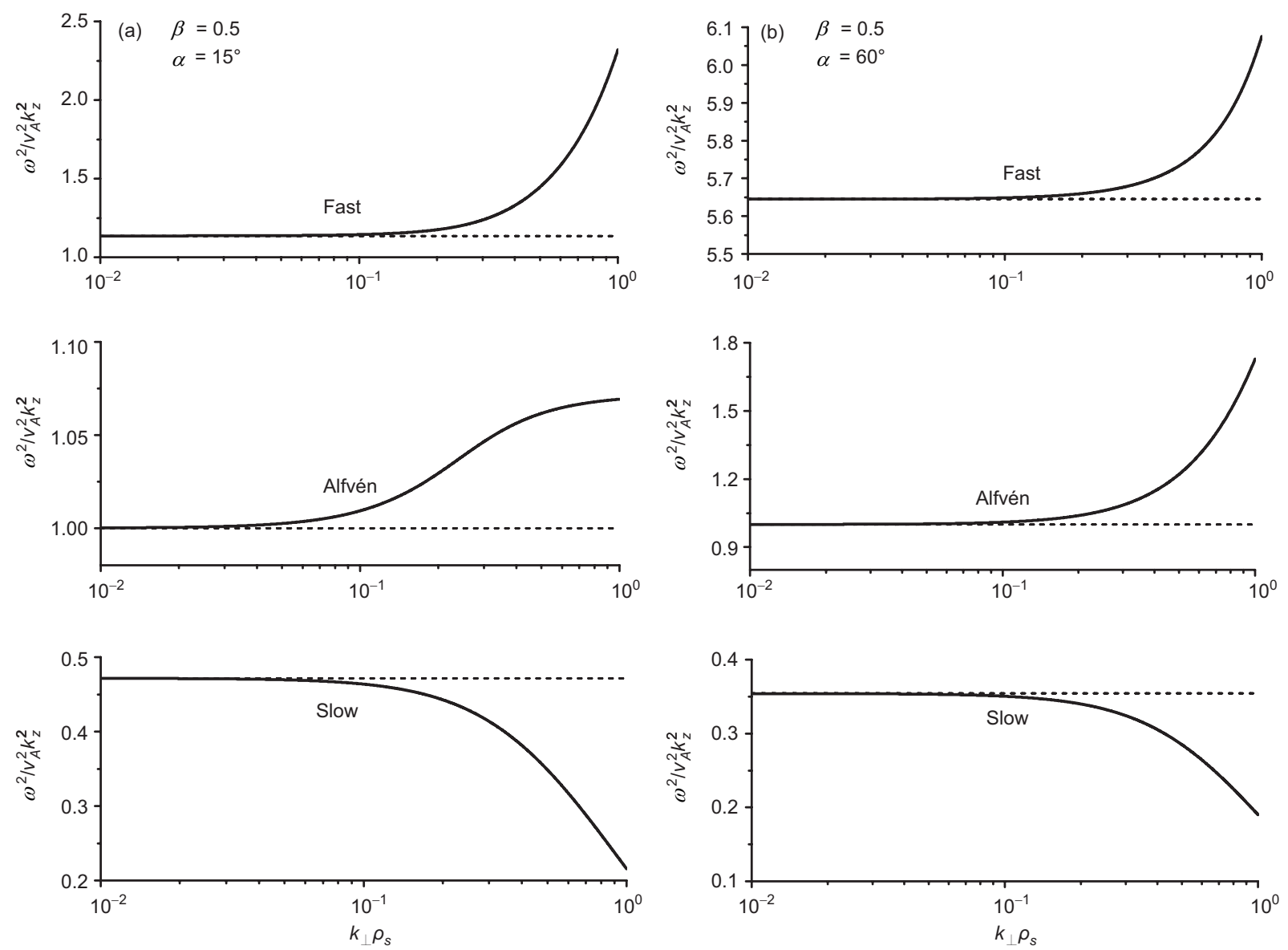

Figure 2 The dispersion relations versus the perpendicular wavenumber. Panels, from the top down, present the fast, Alfvén and slow modes, respectively, where the dashed lines plot the corresponding idea MHD solutions for the sake of comparison and the left and right columns correspond to the cases of quasi-parallel $\left(\alpha=15^{\circ}\right)$ (a) and quasi-perpendicular $\left(\alpha=60^{\circ}\right)$ (b) propagations, respectively.

when $k_{\perp} \rho_{s}<0.1$ and to increase quickly with $k_{\perp} \rho_{s}$ when $k_{\perp} \rho_{s}>0.1$. This clearly implies that the dispersion modification is caused by the short-wavelength effect in the perpendicular direction. The short-wavelength effect leads to the increase of the parallel phase speed for both the fast and Alfvén modes, but the decrease for the slow mode.

On the other hand, the plasma $\beta$ parameter (the ratio of thermal to magnetic pressures) can remarkably influence the dispersion modification as well. Figure 3 shows the dependence of the dispersion relations for the fast, Alfvén and slow modes (from the top down) on the plasma $\beta$ parameter for the quasi-parallel ( $\alpha=15^{\circ}$ for the left column) and quasiperpendicular ( $\alpha=60^{\circ}$ for the right column) propagating cases. We have used the fixed perpendicular wavenumber $k_{\perp} \rho_{s}=0.8$ and have plotted the corresponding ideal MHD solutions with dashed lines for the sake of comparison.

From Figure 3, we find that, for the fast mode, the dispersion modification mainly occurs in the finite $\beta$ regime of $0.001<\beta<1$ for the quasi-parallel propagating case (see the top panel of the left column). For this case, the parallel phase speed is considerably higher than its ideal MHD solution, whereas the modifications are negligibly small for the quasi-perpendicular propagating case (see the top panel of the right column), as shown in the top panel of Figure 1. On the other hand, for the slow mode, the dispersion modification occurs mainly in the high- $\beta$ regime of $0.1<\beta<10$ for both the quasi-parallel and quasi-perpendicular propagating cases, in which the parallel phase speed is lower than its ideal MHD solution (see the bottom panels in Figure 3).

For the Alfvén mode, however, the matter is more complex. In the low $\beta$ regime of $\beta<0.001$, the parallel phase speed is remarkably lower than its ideal MHD solution, but is higher than its ideal MHD solution in the finite- and high- $\beta$ regimes of $0.001<\beta<10$. For the extremely high- $\beta$ regime of $\beta>100$, the parallel phase speed of the Alfvén mode approaches its ideal MHD solution as $\beta$ increases. As shown in Figure 1, the modification in the quasi-perpendicular propagation direction is much larger than that in the quasi-parallel propagation direction in the finite- and high- $\beta$ regimes of $0.001<\beta<10$.

Incidentlally, it is worth noting that, for the quasi-parallel propagating case (see the left column in Figure 3), both the fast and Alfvén modes encounter the Landau resonance of electrons near $\beta \simeq m_{e} / m_{i}<10^{-3}$ due to $v_{A} \simeq v_{T_{e}}$ (the electron thermal speed), where the flow description is invalid and the kinetic theory becomes necessary. 

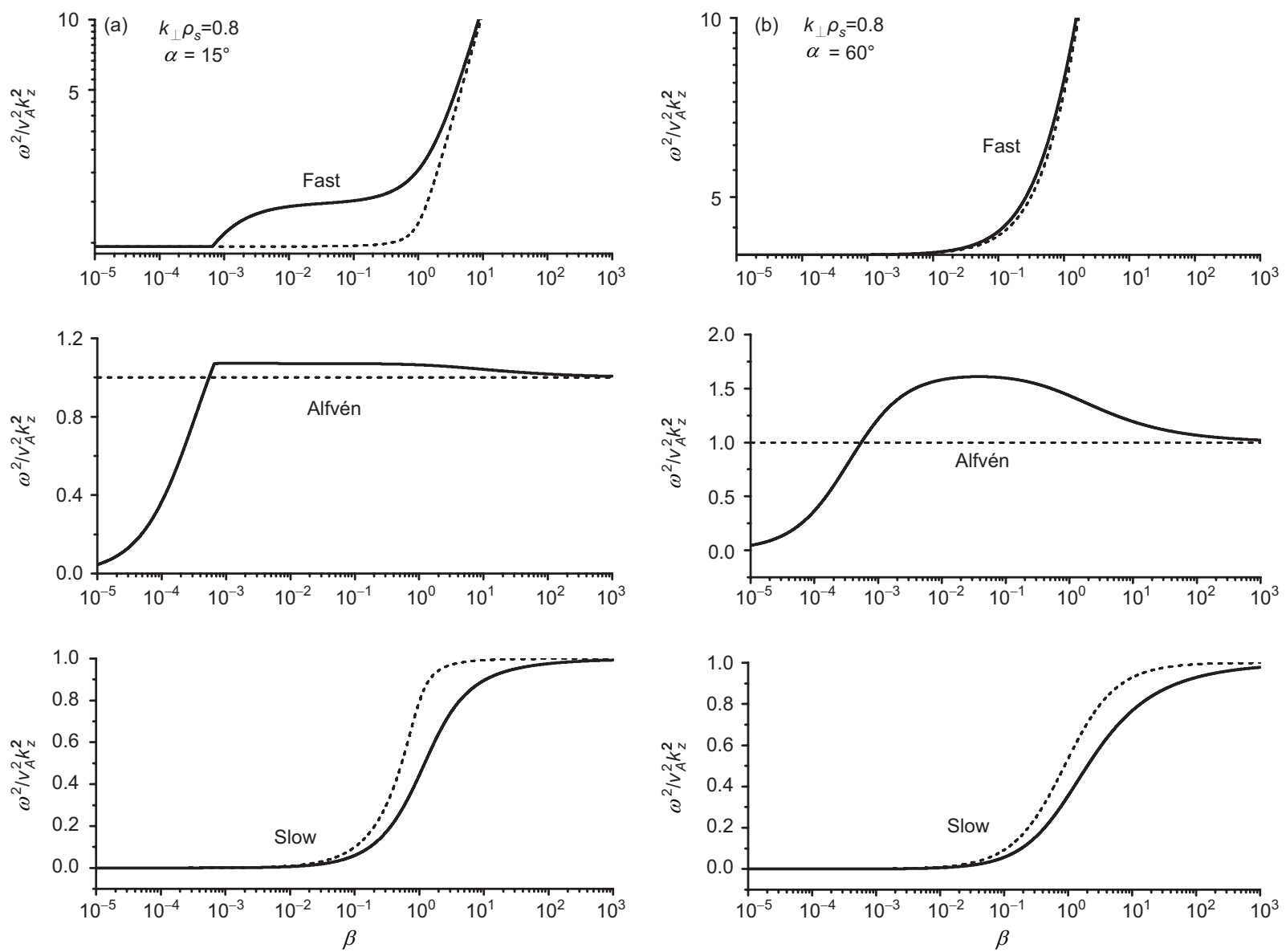

Figure 3 The dispersion relations versus plasma beta parameter. Panels, from the top down, present the fast, Alfvén and slow modes, respectively, where the dashed lines plot the corresponding idea MHD solutions for the sake of comparison and the left and right columns correspond to the cases of quasi-parallel $\left(\alpha=15^{\circ}\right)$ (a) and quasi-perpendicular $\left(\alpha=60^{\circ}\right)$ (b) propagations, respectively.

\section{Low- $\beta$ and quasi-perpendicular propagation limits}

In the low- $\beta$ limit of $\beta \rightarrow 0$, one has approximately, from eq. (14):

$$
A \cong K_{A}+\frac{k^{2}}{k_{z}^{2}}, \quad B \cong K_{A} \frac{k^{2}}{k_{z}^{2}}, \quad C=\frac{\beta}{1+Q+k_{\perp}^{2} \lambda_{e}^{2}} \frac{k^{2}}{k_{z}^{2}} \cong 0 .
$$

Consequently, the dispersion eq. (13) can be approximated as

$$
\Omega^{2}\left(\Omega^{2}-\frac{k^{2}}{k_{z}^{2}}\right)\left(\Omega^{2}-K_{A}\right) \cong \frac{\beta}{1+Q+k_{\perp}^{2} \lambda_{e}^{2}} \frac{k^{2}}{k_{z}^{2}} \cong 0 .
$$

Its three roots are

$$
\Omega_{S}^{2}=0 ; \quad \Omega_{F}^{2}=k^{2} / k_{z}^{2} ; \quad \Omega_{A}^{2}=K_{A} .
$$

This indicates that in the low- $\beta$ limit the slow mode is ordered out and the fast and Alfvén modes are reduced to the compressive Alfvén and the KAW modes, respectively.

On the other hand, in the quasi-perpendicular propagation limit of $k_{\perp}^{2} \gg k_{z}^{2}$ (i.e. $k_{z}^{2} / k^{2} \rightarrow 0$ ), one has approximately, from eq. (14):

$$
\begin{gathered}
A \cong \frac{1+Q+k_{\perp}^{2} \lambda_{e}^{2}+\beta}{1+Q+k_{\perp}^{2} \lambda_{e}^{2}} \frac{k^{2}}{k_{z}^{2}}, \quad B \cong \frac{1+Q+k_{\perp}^{2} \rho_{s}^{2}+2 \beta}{1+Q+k_{\perp}^{2} \lambda_{e}^{2}} \frac{k^{2}}{k_{z}^{2}}, \\
C \cong \frac{\beta}{1+Q+k_{\perp}^{2} \lambda_{e}^{2}} \frac{k^{2}}{k_{z}^{2}} .
\end{gathered}
$$

Consequently, the dispersion eq. (13) can be approximated as

$$
\begin{aligned}
\Omega^{4} & -\frac{1+Q+k_{\perp}^{2} \rho_{s}^{2}+2 \beta}{1+Q+k_{\perp}^{2} \lambda_{e}^{2}+\beta} \Omega^{2}+\frac{\beta}{1+Q+k_{\perp}^{2} \lambda_{e}^{2}+\beta} \\
& \cong \Omega^{6} \frac{1+Q+k_{\perp}^{2} \lambda_{e}^{2}}{1+Q+k_{\perp}^{2} \lambda_{e}^{2}+\beta} \frac{k_{z}^{2}}{k^{2}} \cong 0 .
\end{aligned}
$$

Its two roots,

$$
\begin{aligned}
\Omega_{A, S}^{2}= & \frac{1}{2} \frac{1+Q+k_{\perp}^{2} \rho_{s}^{2}+2 \beta}{1+Q+k_{\perp}^{2} \lambda_{e}^{2}+\beta} \\
& \times\left[1 \pm \sqrt{1-4 \frac{1+Q+k_{\perp}^{2} \lambda_{e}^{2}+\beta}{\left(1+Q+k_{\perp}^{2} \rho_{s}^{2}+2 \beta\right)^{2}} \beta}\right],
\end{aligned}
$$


are the coupling modes of the Alfvén and slow modes. In particular, for the low- $\beta$ limit of $\beta \ll 1$, one has

$$
\Omega_{A}^{2} \cong K_{A} ; \quad \Omega_{S}^{2} \cong \frac{\beta}{1+Q+k_{\perp}^{2} \rho_{s}^{2}+2 \beta} .
$$

This indicates that the two coupling modes are decoupled into the KAW and ion-acoustic modes. The fast mode is ordered out in the quasi-perpendicular propagation limit.

\section{Summary and conclusion}

The MHD (fast, Alfvén and slow) waves are intrinsic and ubiquitous fluctuation processes in space and astrophysical plasmas and play an important role in their dynamical coupling and energy transportation. In particular, it has been widely accepted that the dispersive small-scale MHD waves could be responsible for the dissipation of wave energies, as well as particle acceleration and plasma heating, which occur continually in various eruptive phenomena from terrestrial aurora and solar flares, to cosmic rays. The dispersive small-scale Alfvén wave, called KAW, has been extensively investigated and applied to plasma energization processes [1-4]. In analogous works, however, little attention has been paid to the fast and slow modes. Recent investigations on the MHD turbulence have proposed that the compressible fluctuations in the fast and slow modes, as well as the KAWs, may need to be taken further into consideration when referring to the steeping of the spectrum at higher wavenumbers [17-20,26]. In this paper, the dispersive MHD waves, with the short-wavelength modification, are self-consistently revised on the basis of the two-fluid model. The dispersion equation, including the short-wavelength effect, and its three roots, are presented as the short-wavelength modification versions of the three ideal MHD (fast, Alfvén and slow) modes.

The results show that the fast mode is considerably modified by the short-wavelength effect in the quasi-parallel propagation direction and the parallel phase speed increases by a factor of $\sim 2$ in the quasi-parallel propagation direction of $\alpha<20^{\circ}$. Moreover, the dispersion modification occurs mainly in the finite- $\beta$ regime of $0.001<\beta<1$.

In contrast to the fast mode, the Alfvén mode is modified by the short-wavelength effect primarily in the quasiperpendicular propagation direction and the parallel phase speed increases by a factor of $\sim 1.5$ in the quasi-perpendicular propagation direction of $\alpha>50^{\circ}$. In the low- $\beta$ regime of $\beta<0.001$, the parallel phase speed is remarkably lower than its ideal MHD solution, whereas the parallel phase speed is higher than its ideal MHD solution in the finite- and high- $\beta$ regimes of $0.001<\beta<10$. Furthermore, in the extremely high $\beta$ regime of $\beta>100$, the parallel phase speed of the Alfvén mode approaches its ideal MHD solution.

Different from both the fast and Alfvén modes, the slow mode clearly departs from its ideal MHD solution between $\alpha=0^{\circ}$ and $\alpha=90^{\circ}$, due to the short-wavelength effect.
The parallel phase speed decreases by a factor of $\sim 2$ in the quasi-parallel propagation direction of $\alpha<20^{\circ}$ and a slightly lower factor of $\sim 1.5$ in the quasi-perpendicular propagation direction of $\alpha>50^{\circ}$. Moreover, the dispersion modification occurs predominantly in the high- $\beta$ regime of $0.1<\beta<10$.

Finally, our results show further that, in the low- $\beta$ limit of $\beta \rightarrow 0$, the slow mode is ordered out, the fast mode reduces to the compressive Alfvén mode, and the shear Alfvén mode leads to KAWs. Conversely, in the quasi-perpendicular propagation limit of $k_{\perp}^{2} \gg k_{z}^{2}$, the fast mode is ordered out and the shear Alfvén and slow modes become coupled.

This work was supported by the National Natural Science Foundation of China (10973043, 41074107), and National Basic Research Program of China (2011CB811402).

1 Hasegawa A, Uberoi C. The Alfvén Wave, DOE Critical Review SeriesAdvances in Fusion Science and Engineering, Technical Information Center, U.S. Department of Energy, Washington, DC, 1982

2 Spies G O, Li J. On the kinetic Alfvén wave. Phys Fluids B, 1990, 2 2287-2293

3 Lysak R L, Lotko W. On the kinetic dispersion relation for shear Alfvén waves. J Geophys Res, 1996, 101: 5085-5094

4 Wu D J, Chao J K. Recent progress in nonlinear kinetic Alfvén waves. Nonlinear Proc Geoph, 2004, 11: 631-645

5 Hasegawa A, Chen L. Kinetic processes in plasma heating by resonant mode conversion of Alfvén wave. Phys Fluids, 1976, 19: 1914-1924

6 Ross D W, Chen G L, Mahajan S M. Kinetic description of Alfvén wave heating. Phys Fluids, 1982, 25: 652-677

7 Huang L, Qin X M, Ding N, et al. Heating Finite Beta Tokamak-Plasmas by Alfvén Waves. Chin Phys Lett, 1991, 8: 232-235

8 Wu D J. Model of nonlinear kinetic Alfvén waves with dissipation and acceleration of energetic electrons. Phys Rev E, 2003, 67: 027402

9 Wu D J. Dissipative solitary kinetic Alfvén wave and electron acceleration. Phys Plasmas, 2003, 10: 1364-1370

10 Wu D J, Chao J K. Auroral electron acceleration by dissipative solitary kinetic Alfvén waves. Phys Plasmas, 2003, 10: 3787-3789

11 Wu D J, Chao J K. Model of auroral electron acceleration by dissipative nonlinear inertial Alfvén wave. J Geophys Res, 2004, 109: A06211

12 Wu D J, Fang C. Two-fluid motion of plasma in Alfvén waves and the heating of solar coronal loops. ApJ, 1999, 511: 958-964

$13 \mathrm{Wu}$ D J, Fang C. Coronal plume heating and kinetic dissipation of kinetic Alfvén waves. ApJ, 2003, 596: 656-662

14 Wu D J, Fang C. Sunspot chromospheric heating by kinetic Alfvén waves. ApJ, 2007, 659: L181-L184

15 Wu D J, Yang L. Anisotropic and mass-dependent energization of heavy ions by kinetic Alfvén. Astron Astrophys, 2006, 452: L7-L10

16 Wu D J, Yang L. Nonlinear interaction of minor heavy ions with kinetic Alfvén waves and their anisotropic energization in coronal holes. ApJ, 2007, 659: 1693-1701

17 Goldreich P, Sridhar S. Toward a theory of interstellar turbulence. 2 Strong Alfvénic turbulence. ApJ, 1995, 438: 763-775

18 Goldreich P, Sridhar S. Magnetohydrodynamic turbulence revisited. ApJ, 1997, 485: 680-688

19 Galtier S, Nazarenko S V, Newell A C, et al. A weak turbulence theory for incompressible magnetohydrodynamics. J Plasma Phys, 2000, 63: $447-488$

20 Galtier S, Chandran B. Extended spectral scaling laws for shear-Alfvén wave turbulence. Phys Plasmas, 2006, 13: 114505 
21 Heyvaerts J, Priest E R. Coronal heating by phase-mixed shear Alfvén waves. Astron Astrophys, 1983, 117: 220-234

22 Ofman L, Davila J M. Alfvén wave heating of coronal holes and the relation to the high-speed solar wind. J Geophys Res, 1995, 100: $23413-$ 23425

23 Lee M A, Roberts B. On the behavior of hydromagnetic surface waves. ApJ, 1986, 301: 430-439

24 Hollweg J V. Resonance absorption of magnetohydrodynamic surface waves Physical discussion. ApJ, 1987, 312: 880-885

25 Hollweg J V, Yang G. Resonance absorption of compressible magnetohydrodynamic waves at thin surfaces. J Geophys Res, 1988, 93: 54235436

26 Howes G G, Cowley S C, Dorland W, et al. A model of turbulence in magnetized plasmas: Implications for the dissipation range in the solar wind. J Geophys Res, 2008, 113: A05103

27 Marson J, Goldreich P. Simulations of incompressible magnetohydrodynamic turbulence. ApJ, 2001, 554: 1175-1196

28 Cho J, Lazarian A, Vishniac E T. Simulations of magnetohydrodynamic turbulence in a strongly magnetized medium. ApJ, 2002, 564: 291301

29 Belcher J W, Davis Jr L. Large-amplitude Alfvén waves in the interplanetary medium, 2. J Geophys Res, 1971, 76: 3534-3563

30 Matthaeus W H, Goldstein M L, Roberts D A. Evidence for the presence of quasi-two-dimensional nearly incompressible fluctuations in the solar wind. J Geophys Res, 1990, 95: 20673-20683

31 Wilkinson P N, Narayan R, Spencer R E. The scatter-broadened image of CYGNUS-X-3. Mon Not R Astron Soc, 1994, 269: 67-88

32 Trotter A S, Moran J M, Rodriguez L F. Anisotropic radio scattering of NGC 6334B. ApJ, 1998, 493: 666-679

33 Rickett B J, Kedziora-Chudczer L, Jauncey D L. Interstellar scintillation of the polarized flux density in quasar PKS 0405-385. ApJ, 2002, 581: $103-126$

34 Dennett-Thorpe J, De Bruyn A G. Annual modulation in the scattering of J1819+3845: Peculiar plasma velocity and anisotropy. Astron Astrophys, 2003, 404: 113-132

35 Leamon R J, Smith C W, Ness N F, et al. Observational constraints on the dynamics of the interplanetary magnetic field dissipation range. J Geophys Res, 1998, 103: 4775-4787
36 Leamon R J, Smith C W, Ness N F, et al. Damping of oblique kinetic Alfvén waves as the cause of the interplanetary magnetic field dissipation range. Eos Trans AGU, Spring Meet Suppl, 1998, 79: S272

37 Goertz C K. Kinetic Alfvén waves on auroral field lines. Planet Space Sci, 1984, 32: 1387-1392

38 Hollweg J V. Kinetic Alfvén wave revisited. J Geophys Res, 1999, 104 14811-14819

39 Ren Z P, Wan W X, Wei Y, et al. A theoretical model for mid- and low-latitude ionospheric electric fields in realistic geomagnetic fields. Chinese Sci Bull, 2008, 53: 3883-3890

40 Zhang Y, Chen J Y, Feng X S. Predicting the shock arrival time using 1D-HD solar wind model. Chinese Sci Bull, 2010, 55: 1053-1058

41 Yue C, Zong Q G, Wang Y F. Response of the magnetic field and plasmas at the geosynchronous orbit to interplanetary shock. Chinese Sci Bull, 2009, 54: 4241-4252

42 Yao L, Liu Z X, Zuo P B, et al. Responses of properties in the plasma sheet and at the geosynchronous orbit to interplanetary shock. Chinese Sci Bull, 2009, 54: 3308-3317

43 Sun L P, WU D J, Wang D Y. Suprathermal particle events observed by WIND spacecraft in interplanetary space during 1995-1999 and their classification. Sci China Ser E-Tech Sci, 2008, 51: 1542-1553

44 Zhang X G, Pu Z Y, Ma Z W, et al. Roles of initial current carrier in the distribution of field-aligned current in 3-D Hall MHD simulations. Sci China Ser E-Tech Sci, 2008, 51: 323-336

45 Li X, Weng C S. 2-D viscous magnetohydrodynamics simulation of plasma armatures with the CE/SE method. Chinese Sci Bull, 2009, 54: 1641-1647

46 Lu H Y, Lee C H. Influence of Hall effects on characteristics of magnetohydrodynamic converging channel. Chinese Sci Bull, 2010, 55 1454-1460

47 Lu H Y, Lee C H. Simulation of three-dimensional nonideal MHD flow at low magnetic Reynolds number. Sci China Ser E-Tech Sci, 2009, 52 3690-3697

48 Lu H Y, Lee C H, Dong H T. Characterization of the three-dimensional supersonic flow for the MHD generator. Sci China Ser G-Phys Mech Astron, 2009, 52: 534-545

49 Wu D J. Effects of ion temperature and inertia on kinetic Alfvén waves. Commun Theor Phys, 2003, 39: 457-464

Open Access This article is distributed under the terms of the Creative Commons Attribution License which permits any use, distribution, and reproduction in any medium, provided the original author(s) and source are credited. 